\title{
Factors Affecting Uptake of Covid 19 Vaccines - A Case of Mbala District, Northern Zambia
}

\author{
Daniel Sinkala $^{1 *}$, Ellen Munyati ${ }^{2}$, Karthik, $\mathrm{A}^{1,2}$ \\ ${ }^{1}$ District Health Director, Ministry of Health, Mbala, Zambia \\ ${ }^{2}$ Public Health, College of Nursing, Ndola, Zambia
}

\begin{abstract}
The desire to control Covid 19 pandemic has continued to exist in the Mbala district of Zambia, with the latest trend showing a significant increase in a number of people testing positive, with a corresponding increase in vaccines $(A Z, J J)$ hesitancy resulting in a low $(2.8 \%)$ vaccination rate in the district. Thus, the need to probe further on covert factors under acceptability (myths, AEFs) and accessibility (vaccines availability, adequacy of vaccination sites) that could be reducing Covid 19 vaccine uptake in Mbala district. The study used a cross-sectional survey, a mixed (quantitative \& qualitative) method in eliciting information from data sources covering a period of six months (April - September 2021). In all, 341 research respondents were interviewed through self-administered questionnaires. Data were analysed using descriptive statistics and binary logistic regression under SPSS v16. Study findings provide sufficient evidence that high myth (89.8\%) reduced acceptability levels, while a low number of vaccination sites (59\%) reduced accessibility, resulting in a low uptake rate in Mbala district. Therefore, the study recommended; building up of well-financed District Covid 19 task forces with educational aims on acceptability and accessibility, Governments to introduce specific funding lines for Covid 19 vaccination campaign and enshrine it into monthly grants for routine-outreach Covid 19 vaccination services, and Local Governments through the directorate of Public Health to introduce by-laws on mandatory Covid 19 vaccination passports for the public. With proper implementation of all these study recommendations, Covid 19 vaccination coverage rates can increase drastically across all districts of northern Zambia.
\end{abstract}

Keywords: Acceptability, Accessibility, AEFs, Adequacy, Availability, Covid 19 vaccines, Myth.

\section{Introduction}

Covid 19 cases continue to rise in Mbala district as people increasing Shan vaccination campaign due to acceptability and accessibility factors [1]. The increasing desire of Mbala people to survive socio-economically has seen remarkable cross-border trade between Zambia (Mbala) and Tanzania (Kalambo) districts. This is coupled with the massive intrinsic movement of people in search of basic needs. This has put Mbala district at increased risk of contracting Covid 19 disease at a faster rate [1].

The new coronavirus, classified as a severe acute respiratory syndrome (SARS) - $\mathrm{CoV}-2$ that emerged in Hubei province in China, causes a new coronavirus disease, which was termed Covid-19 by WHO on 11 February 2020 [2]. Covid-19 has claimed more than 4.7 million lives around the world by early September 2021. It is not the first coronavirus, which infects humans; the pathogenic viruses that cause human diseases (human coronaviruses, $\mathrm{HCoV}$ ) include 6 other members designated as SARS-CoV, middle east respiratory syndrome (MERS)-CoV, HCoV-HKU1, HCoV-NL63, HCoV-OC43 and $\mathrm{HCoV}-229 \mathrm{E}$ [2].

Human coronaviruses were first identified in the mid-1960s; they were named for the 
crown-like spikes on their surface. The SARS-CoV-2 virus belongs to $\beta$-coronavirus, which also include MERS-1-CoV, SARS-CoV-1, NCoV-OC43 and HCoV-HKU1. The pathogenesis of Covid 19 follows the primary target cells for SARS-CoV-2 which are epithelial cells of the respiratory and gastrointestinal tract, that contain angiotensin converting enzyme 2 (ACE2), which is utilized by the virus to enter the cell; it is, however, hard to believe that the penetration of the viral agent into the organism is limited only to these tissues [2].

The clinical presentations of Covid-19 range from asymptomatic/mild symptoms to severe illness and mortality [3]. Common symptoms include fever, cough, and shortness of breath manifested as malignant pneumonia; although many patients present neurological symptoms, such as vomiting, dizziness, headache, and delirium [4]. Other symptoms, such as malaise and respiratory distress, have also been reported in several studies. Symptoms may develop 2 days to 2 weeks after exposure to the virus [5]. Covid 19 disease is currently managed both therapeutically and clinically; common drugs used include antibiotics, steroids, vitamin C, and anti-viral drugs [6]. But the best way of managing outbreaks is through primary prevention, which in this case is vaccination of the mass populations.

In response to Covid 19 vaccination urgency, the global world of science has been using two categories of manufacturing vaccines; Viral Vectors (VV) and Messenger RNA (mRNA) vaccines. Viral Vectors (Johnson \& Johnson and AstraZeneca) use a harmless version of a cold virus as a vector to give our cells the instructions they need to make the coronavirus's spike protein. Johnson \& Johnson uses a human adenovirus, or a cold virus, to create its vaccine, while AstraZeneca uses a chimpanzee version [7, 8]. Johnson \& Johnson's is the first singledose vaccine approved in Canada, while AstraZeneca requires two doses. Messenger RNAs (Modena and Pfizer) are a novel technology that essentially teaches our cells how to produce the coronavirus's spike protein. That triggers an immune response if we become infected with the virus in the future. Whereas the viral vectors use another virus to give our cells the info they need to make the spike protein, mRNA dumps the genetic code in directly, without using another virus as a vessel. Pfizer and Modena use synthetically produced mRNA that's packaged in a fat coating.

The mRNA is dumped into the cell when the vaccine is injected into the arm muscle, and it is then translated into protein to make the antibody [7]. All of the approved Covid-19 vaccines train the body to recognize the spike protein that coats the outer surface of the coronavirus. The immune system recognizes the protein and makes antibodies, which then allow us to fend off an attack if exposed in the future. Experts say it takes a couple of weeks for the body to build up some level of immunity with any of the vaccines [8]. All four of the vaccines basically work the same way, but there's one less component involved with the mRNA versions.

Early pitfalls against the mRNA technology were that it was too unstable and fragile, with the mRNA disintegrating upon entering the body. But the problem was solved by packaging it in the fat coating, giving it something to help bind onto cells easier. Thus, the gazetted vaccines in the Mbala district of Zambia are AstraZeneca and Johnson \& Johnson, which are both viral vector vaccines.

However, the Covid 19 vaccines have faced many causes of vaccine hesitancy that include many doubts and concerns related to COVID19 vaccines as well as a diminished level of confidence and trust, by segments of the public in the nation's leaders in government, medical, and business communities, that those groups once enjoyed [7, 8]. Nonetheless, vaccination with COVID-19 vaccines still remains the only way that COVID-19 can be eliminated or at least controlled today, and vaccine hesitancy is 
the potential long-standing rival, an archenemy in this battle.

The present report describes how the allergist/immunologist not only plays a major role in the delivery of specialized therapy of COVID-19 but also in educating the public with regard to the importance of COVID-19 vaccines, in dispelling misinformation, and in promoting trust for vaccine acceptance but must be informed with the most accurate and current information on Covid 19 vaccines available [8].

The problem of covid 19 vaccines uptake is confounded by the continued mystery of understanding the unpredictable nature of covid 19 disease spread and control $[9,10]$. Thus, the purpose of this paper was to highlight the acceptance and accessibility factors associated with vaccines uptake in the Mbala district of Zambia and suggest measures that could be used to address them within the local context.

\section{Significance of the Study}

Information on the effect of Covid 19 vaccines acceptability and accessibility factors (myth, AEFs, vaccines availability, and adequacy of vaccination sites) on vaccines uptake by people of Mbala district is important in containing disease burden, especially in rural areas.

This study will also bridge the scientific knowledge gap and contribute to a paradigm shift in the prevention and management of the Covid 19 pandemic a public health perspective. Lastly, it will contribute to the debate on the increasing number of vaccination sites through Covid 19 vaccination policy by policymakers.

\section{Problem Statement}

The desire to control the Covid 19 pandemic has continued to exist in the Mbala district of Zambia, with the latest trend showing a significant increase in the number of people testing positive for the disease, with an upward prevalence trend of $0.97 \%$ (July), $1.10 \%$ (August), and 1.14\% (September) of 2021 [11]. Because of this, Mbala district joined the national Covid 19 vaccination campaign on 28th April 2021 with the district target population of 29,346 (the above 18 years old). But, after six months of vaccination campaign (by September 2021, only $2.8 \%$ of the target population got fully vaccinated, $53.2 \%$ were still not due for dose 2, while $44 \%$ defaulted due to the increasing number of people sharing to receive Covid 19 vaccines [1, 11]. This is compounded by strong cultural beliefs; "Chibola $=$ male infertility" and "Ukufutikila $=$ steaming in a closed apartment boiling herbs" that negatively affect modern health-seeking behaviour among this Covid 19 vulnerable population, especially men [1]. The male folk believe that when they receive any Covid 19 vaccine, they will develop "secondary male infertility" traditionally known as "Chibola" and also develop covid 19 disease [1, 12]. Many efforts have been tried to sensitize the community on the reliability of Covid 19 vaccines with support from government partners such as World vision Zambia, John Snow Initiative, EQUIP, and Household in Distress, but still the vaccination coverage proves to be very low in the district [1].

Table 1. Prevalence and Full Vaccination Rates over Six Months

\begin{tabular}{|l|l|l|l|l|l|l|}
\hline Months & Apr & May & Jun & Jul & Aug & Sep \\
\hline Prev R & 0.12 & 0.24 & 0.63 & 0.97 & 1.1 & 1.14 \\
\hline Full VR & 0 & 0.21 & 0.42 & 2.62 & 2.65 & 2.8 \\
\hline
\end{tabular}

Although the Covid 19 vaccination campaign has proved to have a paradoxical effect on disease prevalence rate, the uptake still proves to be very low (2.8\% fully vaccinated target population) $[1,11]$.
Thus, the need to probe further on covert factors under acceptability and accessibility that could be reducing Covid 19 vaccine uptake in the Mbala district. 


\section{Materials and Methods}

\section{Research Design and Study Population}

The study used a multi-site cross-sectional (survey) approach that was quantitative and qualitative in nature as data was collected at 28 sites. The primary units of the investigation were the target population of Mbala district. The study population was 3,405 people, with a calculated final sample size of 341 respondents [Eq. 1]. This research design was suitable to meet the objectives of the study and presented both quantitative and qualitative data.

The primary exposure was age 18 years and above, not pregnant and residing in Mbala district [13]. The secondary exposure was being vaccinated with Covid 19 vaccines between April-September 2021. The primary outcome was a list of vaccinated people in Mbala district using gazetted vaccines with $100 \%$ protection for $\mathrm{AZ}$ and $67 \%$ for JJ [14]. While secondary outcomes included: acceptance levels, evaluated using: Johns Hopkins - Covid 19 "Myths" Scale, and Mbala DHO - Covid 19 "AEFs" Scale, and Accessibility levels, evaluated using; PAHO - Covid 19 vaccine "availability" assessment scale, and Ottawa Public Health - Covid 19 Vaccination sites "adequacy" assessment scale.

The study involved a sample of 341 respondents calculated (eq. 1) and selected using simple systematic sampling. Data were collected in the month of September 2021 using; checklist, Line lists, Covid 19 vaccination registers, structured questionnaire, and structured personal interviews. The study used statistical package for social sciences (SPSS) version 16 in analysing data using binary logic regression in which Covid 19 vaccines acceptability and Vaccine accessibility were used as dependent variables while average myth level, average AEFs level, vaccination sites adequacy, and vaccines availability were used as independent (covariates) variables.

\section{Ethical Considerations}

In compliance with the ethical guidelines of the University of Zambia - School of Medicine and ERES Ethics Review Board, the researcher clearly stated the purpose of the research, duration, methods used and data collection instruments, which were approved. Participants' consents were sought before interviews all the time. Interviews were made short in order to preserve time for research respondents.

\section{Sample Size Determination}

The study first used the vaccination coverage formula (4PQ / $\mathrm{D}^{2}$ ) to get a sample frame of 3,405 vaccinated people in the Mbala district. Then simple systematic sampling was used to select every $10^{\text {th }}$ respondent to participate in the study, and this gave a final sample of 341. This meant that participants were selected systematically to participate in the research after accessing sufficient information and an adequate understanding of both the proposed research and the implications of participating in the research. The final sample of research respondents was then subjected to structured personal interviews.

\section{Data Collection}

Data was collected using; a checklist, Line lists, Covid 19 vaccination registers, structured questionnaires, and structured personal interviews. The interview questionnaire was administered during the scheduled visits to all the rural health facilities where sampled respondents came from. The questionnaire targeted factors that affected Covid 19 vaccines uptake: myths, AEFs, vaccines availability, and adequacy of vaccination sites. The questionnaire was in English; thus, research assistants were multilingual with proficiency in Mambwe, Lungu, and Namwanga for easy translation whenever needed. The collected data was cleaned and analysed using descriptive statistics. 
Adjusted Odds and percentages were used to describe the effects of these factors on reducing Covid 19 uptake by people of Mbala district using binary logistic regression model, under statistical package for social sciences (SPSS) version 16.

\section{Results}

The study enrolled a total of 341 respondents, and all of them were reached and data collected from them. The tabular statistical results were presented in logistic regression and Descriptive frequency form.

\section{Descriptive (Frequency) Results:}

Table 2. Myths by Stakeholders on Covid 19 Vaccine Uptake

\begin{tabular}{|l|l|l|l|}
\hline Myth level & Average score & Frequency $(\mathbf{n}=\mathbf{3 4 1})$ & Percent $(\%=\mathbf{~ 1 0 0})$ \\
\hline \multirow{2}{*}{ Mild } & 0.2 & & 4 \\
\cline { 2 - 4 } & 0.4 & 14 & 4.0 \\
\hline Moderate & 0.6 & 17 & 5.0 \\
\hline \multirow{2}{*}{ Severe } & 0.8 & 34 & 10.0 \\
\cline { 2 - 4 } & 1.0 & 272 & 79.8 \\
\hline \multirow{2}{*}{ Totals } & & 341 & 100 \\
\hline
\end{tabular}

Table 3. AEFs on Stakeholders due to Covid 19 Vaccine Uptake

\begin{tabular}{|l|l|l|l|}
\hline AEFs level & Average score & Frequency $(\mathbf{n}=\mathbf{3 4 1})$ & Percent $(\% \mathbf{\%} \mathbf{1 0 0})$ \\
\hline Nil & 0.0 & 338 & 99.1 \\
\hline \multirow{3}{*}{ Mild } & 0.2 & 2 & 0.6 \\
\cline { 2 - 4 } & 0.4 & 1 & 0.3 \\
\hline \multirow{2}{*}{ Moderate } & 0.6 & 0 & 0.0 \\
\hline \multirow{2}{*}{ Severe } & 0.8 & 0 & 0.0 \\
\cline { 2 - 4 } & 1.0 & 0 & 0.0 \\
\hline Totals & & 341 & 100 \\
\hline
\end{tabular}

Table 4. Stakeholders' Opinion on Covid 19 Vaccine Availability

\begin{tabular}{|l|l|l|l|}
\hline Variable (Vaccine stock level) & Average score & Frequency $(\mathbf{n}=\mathbf{3 4 1})$ & Percent $(\%=\mathbf{~ 1 0 0})$ \\
\hline Standard stock - both vaccines stocked & 1.0 & 78 & 22.9 \\
\hline Moderate S/O - one vaccine stocked out & 0.5 & 219 & 64.2 \\
\hline Severe S/O - both vaccines stocked out & 0.0 & 44 & 12.9 \\
\hline Totals & & 341 & 100 \\
\hline
\end{tabular}

Table 5. Stakeholders Opinion on Adequacy of Covid 19 Vaccination Sites

\begin{tabular}{|l|l|l|l|}
\hline Variable (Vaccination sites adequacy) & Average score & Frequency $(\mathbf{n}=\mathbf{3 4 1})$ & Percent $(\%=100)$ \\
\hline Standard & 1.0 & 58 & 17 \\
\hline Moderate substandard & 0.67 & 82 & 24 \\
\hline Severe substandard & 0.33 & 201 & 59 \\
\hline Totals & & 341 & 100 \\
\hline
\end{tabular}


Table 6. Stakeholders' Opinion on Covid 19 Vaccine Accessibility and Acceptability

\begin{tabular}{|l|l|l|l|}
\hline Vaccine variable & Average score & Frequency $(\mathbf{n = 3 4 1})$ & Percent (\%= 100) \\
\hline \multirow{3}{*}{ Acceptability } & Easy accept (1.0) & 67 & 19.6 \\
\cline { 2 - 4 } & $\begin{array}{l}\text { Difficulty accepts } \\
(2.0)\end{array}$ & 273 & 80.4 \\
\hline \multirow{2}{*}{ Total } & 341 & 100 \\
\hline \multirow{2}{*}{ Accessibility } & Easy access (1.0) & 83 & 24.3 \\
\cline { 2 - 4 } & Difficulty access (2.0) & 258 & 75.7 \\
\hline \multicolumn{2}{|l}{ Total } & 341 & 100 \\
\hline
\end{tabular}

Table 7. Crosstabulation of Acceptability and Accessibility of Covid 19 Vaccine by Stakeholders

\begin{tabular}{|l|l|l|l|l|}
\hline \multicolumn{2}{|c|}{} & \multicolumn{2}{|l|}{ Accessibility } & \multirow{2}{*}{ Total } \\
\cline { 3 - 4 } \multicolumn{2}{c|}{} & Easy access & Difficulty access & \\
\hline \multirow{2}{*}{ Acceptability } & Easily accepted & $11(3.2 \%)$ & $56(16.4 \%)$ & $67(19.6 \%)$ \\
\cline { 2 - 4 } & Difficulty accepting & $72(21.1 \%)$ & $202(59.2 \%)$ & $274(80.4 \%)$ \\
\hline \multirow{2}{*}{ Total } & $83(24.3 \%)$ & $258(75.7 \%)$ & $341(100 \%)$ \\
\hline
\end{tabular}

\section{Logistic Regression Results}

Objective 1: To determine whether covid 19 vaccines acceptability factors (myths and AEFs) are associated with reduced vaccine uptake by people of Mbala district.

Hypothesis 1: The study rejects the Null Hypothesis (H0) that "none" of the covid 19 vaccines acceptability factors (myth \& AEFs) are associated with reduced vaccine uptake by people of Mbala district and accepts the
Alternate Hypothesis (H1) that at "least one" of the covid 19 vaccines acceptability factors (myth \& AEFs) is associated with reduced vaccine uptake by people of Mbala district, because the adjusted odds ratio produced by binary logistic regression model for myth [0.083 (CI 95\%, $0.008-0.900, \mathrm{p}<0.05)]$ is statistically significant at $95 \%$ confidence level.

Table 8. Binary logistic regression of Acceptability * Myths \& AEFs

\begin{tabular}{|c|c|c|c|c|c|c|c|c|}
\hline \multirow[t]{2}{*}{ Variables } & \multirow[t]{2}{*}{ B } & \multirow[t]{2}{*}{ SE } & \multirow[t]{2}{*}{ Wald } & \multirow[t]{2}{*}{ Df } & \multirow[t]{2}{*}{ Sig. } & \multirow[t]{2}{*}{$\operatorname{Exp}(B)$} & \multicolumn{2}{|c|}{$95 \% \mathrm{CI}$ for $\operatorname{EXP}(\mathrm{B})$} \\
\hline & & & & & & & Upper & Lower \\
\hline Myth & -2.488 & 1.216 & 4.191 & 1 & .041 & .083 & .008 & .902 \\
\hline AEFs & 94.205 & 9.157 & 0.000 & 1 & .999 & 8.179 & .000 & 4.000 \\
\hline Constant & 3.7 .65 & 1.179 & 10.204 & 1 & .001 & 43.143 & & \\
\hline
\end{tabular}

\section{Results 1}

1. Myth - Adjusted Odds Ratio 0.083, CI $0.008-0.900$, statistically significant at $95 \% \mathrm{CL}, \mathrm{p}=0.041$.

2. AEFs - Adjusted Odds Ratio 8.179, CI $0.000-4.000$, statistically insignificant at $95 \% \mathrm{CL}, \mathrm{p}=0.999$.

Objective 2: To determine whether covid 19 vaccines accessibility factors (vaccination sites adequacy \& vaccines availability) are associated with reduced vaccine uptake by people of Mbala district.

Hypothesis 2: The study rejects the Null Hypothesis (H0) that "none" of the covid 19 vaccines accessibility factors (vaccination sites adequacy \& vaccines availability) is associated with reduced vaccine uptake by people of Mbala district and accepts the Alternate Hypothesis (H1) that at "least one" of the covid 19 vaccines accessibility factors (vaccination sites adequacy \& vaccines availability) is 
associated with reduced vaccine uptake by people of Mbala district because the adjusted odds ratio produced by binary logistic regression model for adequacy of vaccination sites $[0.342(\mathrm{CI} 95 \%, 0.135-0.867, \mathrm{p}<0.05)]$ is statistically significant at $95 \%$ confidence level.

Table 9. Binary logistic regression of Accessibility * Vaccine availability \& Vaccination sites adequacy Variables in the Equation

\begin{tabular}{|l|l|l|l|l|l|l|l|l|}
\hline Variables & B & SE & Wald & Df & Sig. & $\operatorname{Exp}(\mathbf{B})$ & \multicolumn{2}{|l|}{ 95\% CI for EXP(B) } \\
\cline { 6 - 9 } & & & & & & & Upper & Lower \\
\hline Vac. $\boldsymbol{A} \boldsymbol{v}$ & -0.061 & .431 & .020 & 1 & .887 & .941 & .405 & 2.188 \\
\hline VS. $\boldsymbol{A d q}$ & -1.072 & .474 & 5.115 & 1 & .024 & .342 & .135 & .867 \\
\hline Constant & 1.716 & .294 & 33.978 & 1 & .000 & 5.564 & & \\
\hline
\end{tabular}

\section{Results 2}

3. Vaccine's availability - Adjusted Odds Ratio 0.941, CI 90.405 - 2.188, statistically insignificant at $95 \% \mathrm{CL}, \mathrm{p}=$ 0.887.

4. Vaccination sites adequacy - Adjusted Odds Ratio 0.342, CI 0.135 - 0.867, statistically significant at $95 \% \mathrm{CL}, \mathrm{p}=$ 0.024 .

\section{Findings}

\section{Descriptive Findings}

1. High rate of severe myth level against Covid 19 vaccines of $89.8 \%$ (306).

2. The study found that $99.1 \%$ (338) reported no complication after receiving the vaccines.

3. Stakeholders' opinion on the availability of vaccines (AZ \& JJ) found that $64.2 \%$ (219) were given no option of choosing the Vaccine they wanted, and most of them received AstraZeneca vaccine.

4. Stakeholders' opinion on the adequacy of vaccination sites found that 59\% (201) reported walking too long distances for them to access the Covid 19 vaccines due to highly spaced vaccination sites in the district.

\section{Logistic Regression Findings}

1. Acceptability factor (myth) is statistically associated with reduced Covid 19 vaccines uptake by people of Mbala district.
2. Accessibility factor (adequacy of vaccination sites) was found to be statistically associated with reduced Covid 19 vaccines uptake in resource-limited areas like Mbala district of Zambia.

\section{Discussion}

This research study found that out of 341 vaccinated people, when interviewed, a total of 83 had easy access to both Covid 19 vaccines and vaccination sites where they received the vaccination services. However, 72 out of 83 had difficulties in understanding and accepting the Covid 19 vaccines (AstraZeneca or Johnson \& Johnson). The descriptive findings also showed that of the vaccinated stakeholders, 202 (59.2\%) had difficulty both accepting and accessing Covid 19 vaccines in the Mbala district. While only 11 (3.2\%) reported easy acceptability and accessibility of the vaccines. 274 (80.4\%) stakeholders' opinions had difficulties in accepting to be vaccinated, while $258(75.7 \%)$ had difficulties in accessing the vaccination services due to a limited number of vaccination points. Therefore, "Acceptability" (myth) was found to be significantly and independently related to reduced uptake of Covid 19 vaccines with odds of 0.083 (CI 95\%, $0.008-0.900, p<$ P) among vaccinated people. Of the five myths that were considered in this study (Young adults don't need to get vaccinated, Vaccine will cause Covid disease, Vaccine is unsafe as it was rushed production, Vaccine causes severe side effects, and Vaccine 
is a microchip that causes infertility = "Chibola"), Chibola was the most common and highly feared by stakeholders. They believed that if this happened then, they could lose societal dignity and self-esteem. This high rate of severe myth level against Covid 19 vaccines of $89.8 \%$ (306) could have been the major contributor to the majority of eligible people in Mbala district shaning to accept the Covid 19 vaccines. This is consistent with findings by other scholars that "a proper behavior by the public towards vaccination starts with trusting the vaccines first [10]. Trusting medicine should be a common positive feeling when an infectious disease has become pandemic. Usually, a patient does not exhibit such burden of doubts and refusal against a simple well understood therapeutic drug, despite knowing adverse effects. The atavistic fear against vaccines dates back to Jenner in the XVIII century [7]. Therefore, as people get convinced about Covid 19 vaccines as a source of "modified" harmful pathogens, the concern of public vaccination is still far to be fully accepted and, as such, requires a lot of reassurance by medical experts to the public [15]. Thus, the scientific community must expose any critical comment, in the highest proactive way, in order to expand a debate that will ensure transparency of these Covid 19 vaccines from manufacturing companies and ensure citizens and public opinion [16, 17]. If we altogether trust these fundamental keystones, then anyone can directly accept the vaccines and contribute to rendering our resource-limited areas that are more vulnerable to subsequent Covid 19 pandemic waves pleasant and desirable [7]. Because of this rampant vaccine hesitancy by the general public, it is inevitable to inform authorities to balance voluntary and mandatory scales of issuance of "Vaccination passports" to the general population that receive Covid 19 vaccines, and they must be available and accessible to all to prevent exacerbating existing societal inequalities and worsening the health divide. The incorporation of this vaccination campaign into routine vaccinations can go a long way in achieving public herd immunity against this pandemic disease. This is consistent with findings by other scholars that scarcity of vaccines and an unequal distribution of vaccines globally and within nations coupled with a lot of uncertainties surrounding Covid 19 vaccines clinical trials, especially on special groups like pregnant women and ill people [18, 19]. Thus, Covid 19 vaccines hesitancy is more likely to be severe in ethnic minorities of black Africans [18, 20]. "Accessibility" (adequacy of vaccination sites) was found to be statistically associated with reduced Covid 19 vaccines uptake in resource-limited areas with odds of 0.342 (CI 95\%, $0.135-0.867, \mathrm{p}<\mathrm{P}$ ) among respondents. Stakeholders' opinion on the adequacy of vaccination sites found that 59\% (201) reported walking too long distances for them to access the Covid 19 vaccines due to highly spaced vaccination sites in the district. The study also found that of the three vaccination siting parameters considered in this study; [standard $(<5 \mathrm{Km})$, substandard (5$10 \mathrm{Km})$, and severe substandard $(10 \mathrm{Km}<)]$, "severe substandard" was the most rampant and most of the stakeholders complained of getting tired and losing a lot of time almost the entire day just in trying to get vaccinated. This also could be a good reason of the target population failing to access the Covid 19 vaccination services, especially the disabled and people with a lot of socio-economic commitments. The majority of these study respondents used walking as means of getting to the vaccination sites. Thus, the six Covid 19 vaccination sites in Mbala district proved not to be adequate according to stakeholders' opinion, which contributed to reduced uptake of the vaccine, especially by those staying more than 10 kilometers from selected vaccination sites.

This is consistent with findings by other scholars that GAVI (COVAX) stressed concern over inequities in the distribution of both vaccination sites and vaccines especially in low-income countries [15, 19]. While 
recognizing the need for additional doses to protect certain vulnerable, immunecompromised populations, the IAVG suggests countries to collect and review more evidence before implementing policies regarding the equitable distribution of both Covid 19 vaccines and vaccination sites for the benefit of the global population [15]. Therefore, districts must be able to access funding for vaccination campaigns of Covid 19 from cooperating partners such as the World Bank and other multilateral development banks. Additional funding for this Covid 19 pandemic vaccination services should also be sought from third-party actors (NGOs and civil society) willing to support districts in increasing vaccination sites through infrastructure, equipment (cold \& supply chain maintenance), transport, and human resource development. This can really help many developing nations like Zambia, where Primary health care is still undergoing a rapid transformation with an aim toward implementing Sustainable Development Goals and advancing universal health coverage under a lot of challenges such as inequitable access and unaffordability of quality primary health care services [21].

\section{Equations}

$$
E S_{209_{6}}=\left(4^{*} P^{*} Q / D 2\right) /(1-0.2)
$$

\section{Conclusion}

This study examined the factors affecting Covid 19 vaccine uptake in the Mbala district of northern Zambia, using a cross-section survey approach that was both quantitative and qualitative in nature. Findings indicate; severe myth level (89.8\%) and severe inadequate (substandard) distribution of vaccination sites $(59 \%)$ in the district. These were the major contributors of "low acceptance" and "low accessibility" levels of Covid 19 vaccines by people resulting in very low vaccines $(\mathrm{AZ} \& \mathrm{JJ})$ uptake in the Mbala district of Zambia. The Outcry against vaccination, held by "scared" people, who were already stressed by pandemic caused fears, maybe a real concern for any vaccination campaign, particularly if associated with long-lasting hesitant and refusal attitudes of some individuals towards vaccination [7]. Therefore, experts of public health must be encouraged, for ethical reasons, to build up a well-financed District Covid 19 task force with educational aims to make people aware of what vaccination may hold for human herd immunity in the COVID-19 era. Governments should, therefore, introduce specific funding lines for Covid 19 vaccination campaign and enshrine it into monthly grants (Recurrent Departmental Charges-RDC) to support the routine Covid 19 vaccination services in all the districts of Zambia. It is high time Local Governments through the directorate of Public Health introduced by-laws on "mandatory Covid 19 vaccination immunity passports" for the general public starting with traders, religious leaders, travellers, and other people who are at high risk of contracting the disease. While the other population can be vaccinated under the routine Covid 19 vaccination programme. With proper implementation of these study recommendations, Covid 19 vaccination coverage rates can increase across all districts of Zambia drastically.

\section{Research Funding}

There was not any external funding for this study. The Principal Investigator covered all the costs needed by the study.

\section{Acknowledgements}

I take this opportunity to thank the Research Committee members that worked tirelessly: Dr Ellen Munyati, Mr. Godfrey Sichali, and Mr. Sokoni Chilando, Mrs. Beatrice Tembo, Mrs. Helen Chinkumba, Mr. Erick Banda, Mr. Helbine Simutowe.

I extend my gratitude to Thesis supervisors/ guides Dr Ellen Milimo Munyati (external) and Ms. R Kousalya (internal).

I also thank members of the Thesis guidelines formulation committee and the 
research scholars who helped in preparing this guideline at Texila American University on time in this challenging period of the Covid 19 pandemic.

I thank the Provincial Health Director, Dr. Lawrence Phiri, for granting me the authority to pursue a Doctorate degree in Public Health and for allowing me to conduct a Thesis in the Northern Province of Zambia.

I extend my sincere thanks to my beloved wife Christabel Mwitwa, my three daughters

\section{References}

[1] Mbala HIMS. (2021). District Health Information Management System. Mbala district health office.

[2] Luca Steardo, Luca Steardo Jr. Et al. (2020). Neuroinfection may contribute to the pathophysiology and clinical manifestations of COVID-19: PMC, Public Health Emergency. Acta Physiol (Oxf). 2020 Apr 11: e13473. Doi: 10.1111/apha.13473 [Epub ahead of print].

[3] Guan W-j, Ni Z-y, et al. (2020). Clinical Characteristics of Coronavirus Disease 2019 in China. New England Journal of Medicine: NEJMoa2002032-NEJMoa

https://www.nejm.org/doi/full/10.1056/nejmoa2002

032.

[4] Chen N, Zhou M, et al. (2020). Epidemiological and clinical characteristics of 99 cases of 2019 novel coronavirus pneumonia in Wuhan, China: a descriptive study. The Lancet. 2020; 395 (10223):507.

https://pubmed.ncbi.nlm.nih.gov/32007143/.

[5] Wang D, Hu B, et al. (2020). Clinical Characteristics of 138 Hospitalized Patients with 2019 Novel Coronavirus-Infected Pneumonia in Wuhan, China. JAMA: 323(11):1061. https://www.ncbi.nlm.nih.gov/pmc/articles/PMC704 $2881 /$.

[6] David J Cennimo. (2021). Coronavirus Disease 2019 (COVID-19) Clinical Presentation: Infectious Diseases. Medscape.

https://emedicine.medscape.com/article/2500114clinical.
(Salifyanji, Mwizukanji, \& Sankananji) and my son (Suwilanji Daniel Jr.) for according to me the much-needed social family support during thesis preparation through to completion.

Last but not the least, I thank all members of Mbala DHO, for the support rendered to the research team throughout the thesis process.

\section{Competing Interests}

The authors declare no competing interests for this manuscript.

[7] Chirumbolo. S. (2021). Vaccination hesitancy and the "myth" on mRNA-based vaccines in Italy in the COVID-19 era: Does urgency meet major safety criteria? Journal of Medical Virology. https://doi.org/10.1002/jmv.26922.

[8] Melissa Couto Zuber. (2021). How J\&J and AstraZeneca differ from the mRNA vaccines from Pfizer and Moderna. The Canadian Press. https://www.bnnbloomberg.ca/how-j-j-andastrazeneca-differ-from-the-mrna-vaccines-frompfizer-and-moderna-1.1589765.

[9] Lauer SA, Grantz KH, Bi Q, et al. (2020). The Incubation Period of Coronavirus Disease 2019 (COVID-19) From Publicly Reported Confirmed Cases: Estimation and Application. Annals of internal medicine.

https://www.acpjournals.org/doi/10.7326/m20-0504. [10] Amanna I, Slifka MK. (2005). Public fear of vaccination: separating fact from fiction. Viral Immunol; $\quad$ 18(2): 315. https://pubmed.ncbi.nlm.nih.gov/16035942/.

[11]Mbala IDSR. (2021). Incidence Disease Surveillance Report. DHO. Pg. 8-20.

[12]Nature News. (2021). Trial hints that Pfizer vaccine could curb covid transmission. www.nature.com/articles/d41586-020-00502-w.

[13]Centers for Disease Control and Prevention. (2021). Vaccination considerations for people who are pregnant. www.cdc.gov/coronavirus/2019ncov/vaccines/recommendations/pregnancy.html.

[14] AstraZeneca. (2021). Covid-19 vaccine AstraZeneca confirms $100 \%$ protection against severe disease, hospitalization, and death in the 
primary analysis of phase III trials. www.astrazeneca.com/media-centre/pressreleases/2021/Covid-19-vaccine-astrazenecaconfirms-protection-against-severe-diseasehospitalisation-and-death-in-the-primary-analysisof-phase-iii-trials.html.

[15] WHO. (Sept. 2021). What Needs to Change to Enhance Covid-19 Vaccine Access: Statement from the Independent Allocation of Vaccines Group of COVAX. https://www.who.int/news/item/24-092021-what-needs-to-change-to-enhance-Covid-19vaccine-access.

[16] Kaddar M. (2020). Economic characteristics of vaccines and immunizations. World Health Organization.

www.who.int/influenza_vaccines_plan/resources/ses sion_2_kaddar.pdf.

[17] Nuffield Council on Bioethics. (2020). Rapid policy briefing: Covid-19 antibody testing and "immunity certification" www.nuffieldbioethics.org/publications/Covid-19antibody-testing-and-immunity-certification.
[18] Hassoun N. (2021). How to make "immunity passports" more ethical. Scientific American. www.scientificamerican.com/article/how-to-makeimmunity-passports-more-ethical.

[19]European Patient Forum, Editorial. (2021). Covid-19 vaccine passports: access, equity, and ethics. $B M J$ https://doi.org/10.1136/bmj.

[20] Scientific Advisory Group for Emergencies. (2021). Factors influencing Covid-19 vaccine uptake among minority ethnic groups. https://assets.publishing.service.gov.uk/government/ uploads/system/uploads/attachment_data/file/95271 6/s0979-factors-influencing-vaccine-uptakeminority-ethnic-groups.pdf.

[21] Sinkala. D. (2021). Factors associated with the distribution of Preeclampsia and Eclampsia among Rural and Urban Women in Childbearing Age - A case of Mbala General Hospital, Northern Province, Zambia. Texila International Journal of Public Health. DOI No. 10.21522/TIJPH.2013.09.03. Art008. https://www.texilajournal.com/publichealth/article/1912-factors-associated-with. 